\title{
Natural History of Borderline Hypertension in a Community
}

- An Epidemiological Aspect -

\author{
Kazuo Ueda, M.D., Teruo Omae, M.D. \\ ICHIRo FujII, M.D., AND TOSHIRo YANAI, M.D.
}

\begin{abstract}
The natural history of borderline hypertension was studied in a general population sample of 1,621 Hisayama residents aged 40 or over, followed for 18 years. The causes of death during the study were verified by postmortem examination in most of the cases. The prevalence of borderline hypertension determined by a single casual blood pressure was almost equivalent at the 3 different cross-sectional surveys $(1961,1973-74,1978)$ accounting for approximately $20 \%$ of the population. Prospectively, "borderline hypertension" was associated with an intermediate risk of cardiovascular mortality between normotensives and hypertensives. Borderline hypertensives with hypertensive organ damage manifested by left ventricular hypertrophy on electrocardiogram, retinal arterial changes and/or proteinuria, were much more related to cardiovascular deaths than those without. Blood pressure (BP) in persons not prescribed antihypertensive medications was examined at 3- or 6-year intervals during the follow up period, and the relationship of long-term changes in BP to subsequent cardiovascular mortality was determined. After adjusting for age, an elevation of BP to the hypertensive range from borderline was significantly associated with subsequent cardiovascular catastrophe, and a perspective labile BP was less related to that. The later evidence of hypertensive BP values in borderline was strongly correlated with initial BP levels in both systolic and diastolic, and also with old-age at the time of entry.

It should also be emphasized that borderline hypertension was much more common than severely hypertensive disease in this community. If control of BP in this category is successful, a large number of cardiovascular deaths can hopefully be prevented, because the population-attributable risk of borderline hypertension is high.
\end{abstract}

B ORDERLINE hypertension is best defined by setting the limits for normotension and hypertension and by interpolating a boundary zone between the two. However, the definition of what is normal and abnormal blood pressure

Key Words:

Borderline hypertension

Natural history

Risk factors

Cardiovascular disease

Variation in blood pressure differs from study to study. The situation becomes more confused when readings oscillat between the entirely normal and quite abnorma range. The World Health Organization orginall: defined borderline hypertension as a single sittin or recumbent blood pressure (BP) being in th, range of systolic BP between 160 and $140 \mathrm{mmH}_{\xi}$ and/or of diastolic BP between 95 and 90 . Therefore, it is worth realizing what characteristics the individuals with this range of BP have

The Second Department of Internal Medicine, Faculty of Medicine, Kyushu University, Fukuoka, Japan

Mailing address: Kazuo Ueda, M.D., The Second Department of Internal Medicine, Faculty of Medicine, Kyushu University, 3-1-1 Maidashi, Higashi-ku, Fukuoka 812, Japan 


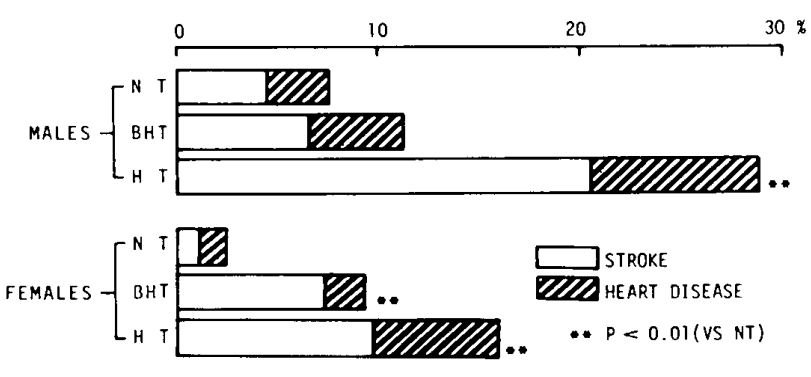

Fig.1. Age-adjusted mortality from cardiovascular disease by blood pressure groups (WHO) in Hisayama, 1961-76.

in the physiologic and epidemiologic aspects.

On the other hand, it has been recognized that elevated blood pressure is associated with increased risks for cardiovascular morbidity and mortality. From the view of community medicine, the high risk associated with high pressure could arise not from a relatively few severe cases but from the very large number of people with mildly raised pressure. There has been, however, little information on the therapeutic guidelines of this condition. The management and treatment of borderline hypertension must be based on a knowledge about the natural history of this disorder.

This paper will describe the natural history of borderline hypertension defined by WHO in respect to whether blood pressure in this range carries an increased risk for future established hypertension and whether cardiovascular mortality in borderline cases is overwhelming. The results have been obtained from an 18-year observation of a cohort of an adult general population in Hisayama town, where a postmor- tem examination has been performed in almost all cases when the subjects died.

\section{STUDY POPULATION AND METHODS}

The Hisayama study is a long-term prospective investigation of the natural history, epidemiology and etiology of the adult cardiovascular diseases in the Japanese general population. The design of the study, methodology, and primary results have been descrived previously. ${ }^{2-4}$ In summary, the cohort consisted of 1,621 healthy Hisayama residents of both sexes aged 40 or over. The baseline data on all relevant variables including measurements of blood pressure was collected from an initial survey in 1961. Medical information and examinations provided evidence that they had no clinical manifestation of cardiovascular diseases at the time of entry. Since then, they have been followed by biennial examinations and causes of death were examined by autopsy in more than $80 \%$ of the subjects who died during the study. The observation period defined here is from November 1, 1961 to October 31, 1979. Cardiovascular disease included all types of cerebrovascular diseases and heart diseases.

Blood pressure was measured several times in both supine and sitting positions using a standard sphygmomanometer. Borderline hypertension was defined as systolic blood pressure of 140 $159 \mathrm{mmHg}$ and/or diastolic blood pressure of 90-94 mmHg. A blood pressure variation in the number of measurements on one occasion and a variation in the length of the observation period between individuals are problems when an effect of blood pressure on subsequent cardiovascular

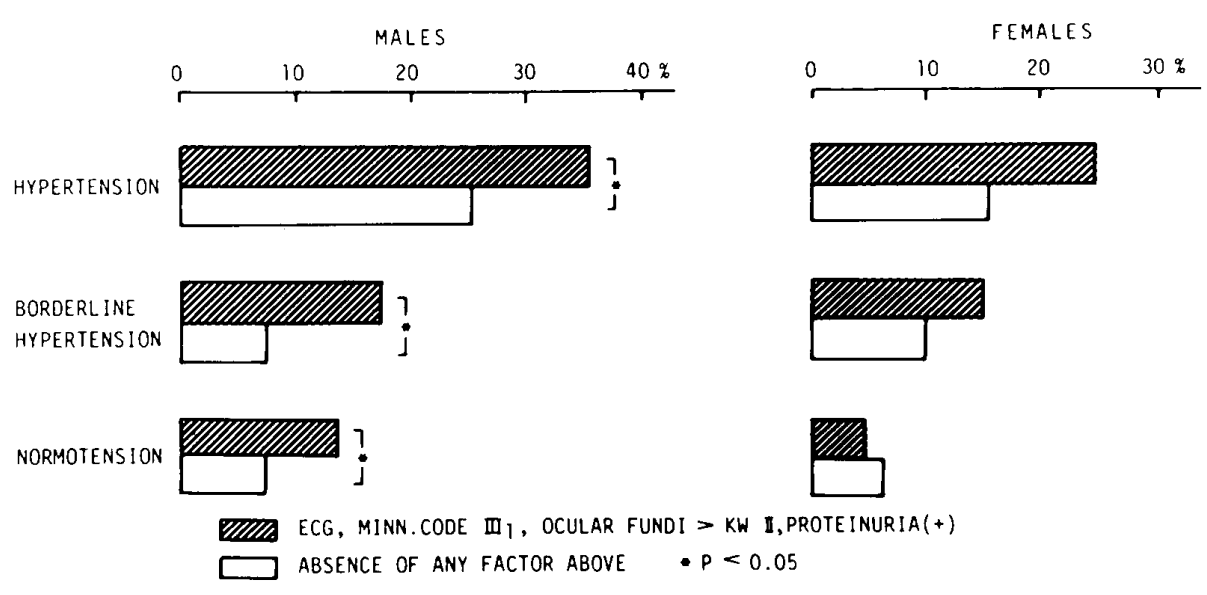

Fig.2. Cardiovascular mortality according to blood pressure groups and associated factors in Hisayama, 1961-79. 
disease is estimated in the longitudinal study. In order to deal with these problems both single and average values of 3 readings of blood pressure at the time of entry were analyzed in relation to cardiovascular mortality. In addition, a change in blood pressure of each subject was observed during the early 6-year period of follow-up and the relationship between the changing pattern of $\mathrm{BP}$ and cardiovascular deaths was evaluated in the following 12-year interval. Persons prescribed antihypertensive medications were excluded only from analysis of changing pattern of blood pressure.

\section{RESULTS \\ Prevalence of Borderline Hypertension}

Table I presents the prevalence of borderline hypertension at 3 different examinations in $1961,1973-74$ and 1978. The screened population was $1,621,2,135$ and 2,449 males and females aged 40 or over, respectively. The prevalence rate of borderline hypertension after age-adjustment was in the range of 20.1 to $23.8 \%$ of the population, and almost equivalent irrespective of sex or time of examination. On the other hand, the prevalence of hypertension showed a decreasing trend among the Hisayama adult population in recent years. It may be considered that management of hypertension is more successful among recent population?

\section{Mortality from Cardiovascular Disease according to Blood Pressure Level at the Time of Entry}

Age-adjusted mortality from cardiovascular disease in the groups classified according to sex and blood pressure at the time of entry is illustrated in Fig. 1. The highest mortality was observed in hypertensives from both sexes, followed by borderline hypertensives and normotensives. Blood pressure variation in each individual related more closely to death from stroke than to that from heart disease. As compared to the subjects with hypertension or normotension, borderline hypertensives had an intermediate risk of cardiovascular death, and mortality in females with borderline hypertension was clearly higher than those with normotension.

Equally important is the risk attributable to different ranges of blood pressure. The ratio of death rates between normotensives and hypertensives was assessed by relative risk, excess risk and population-attributable risk after age-adjust-
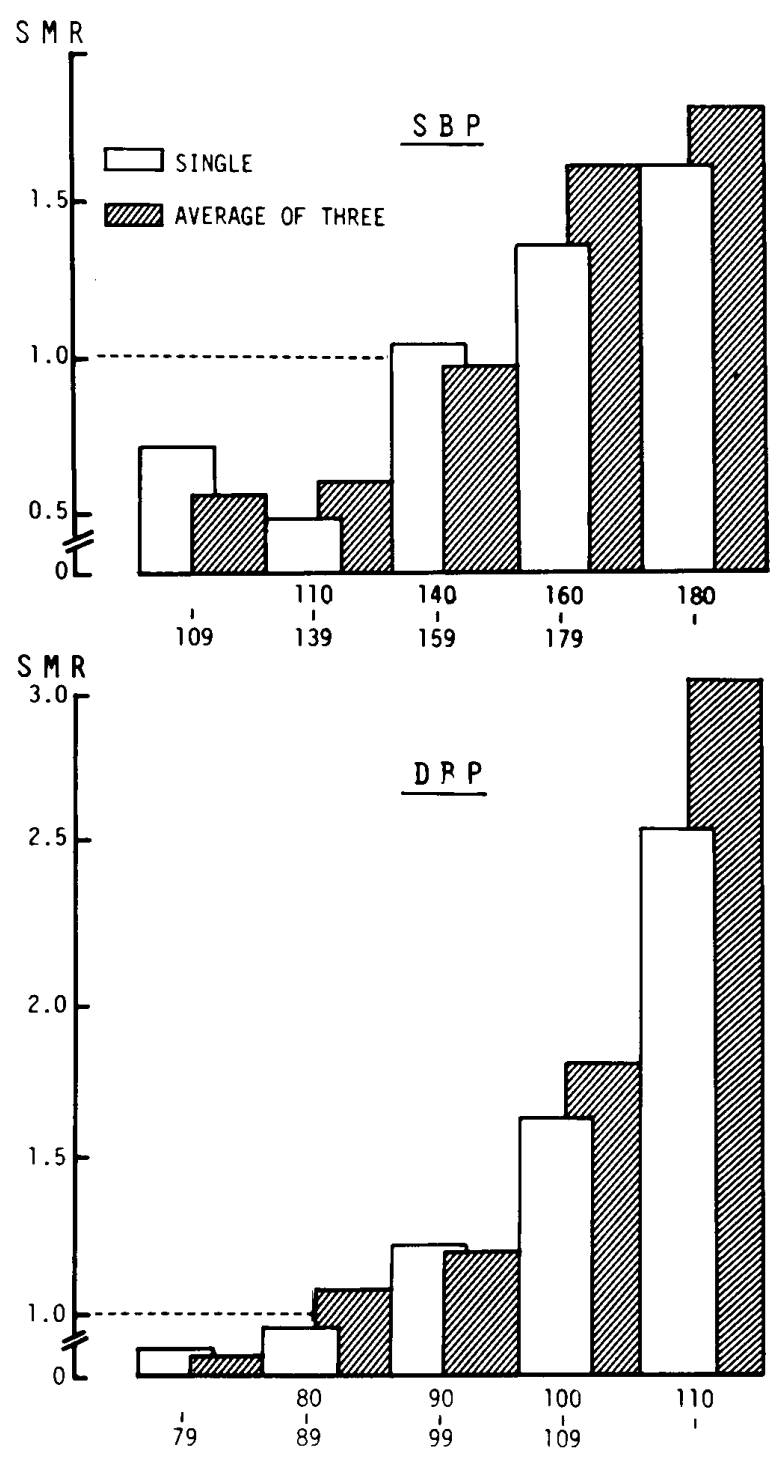

Fig.3. Standardized mortality ratio for cardiovascular disease by blood pressure level, females, in Hisayama, 1961-76. SBF = systolic blood pressure; $\quad \mathrm{DBF}=$ diastolic blood pressure

ment. The population-attributable risk indicates not only the individual risk of death but the frequency of risk at some blood pressure level in the whole community6 It may also be considered a theoretical improvement in the average death rate of the population if hypertension should be eliminated from the population. By multiplying the prevalence by excess mortality, a total of 12.0 deaths per 1000 from cardiovascular disease was calculated to occur in females with borderline hypertension in this community. Sequentially to diastolic hypertensives, this figure was more prominent than that of systolic hypertensives in females. Thus, in this community borderline hypertension in females is 


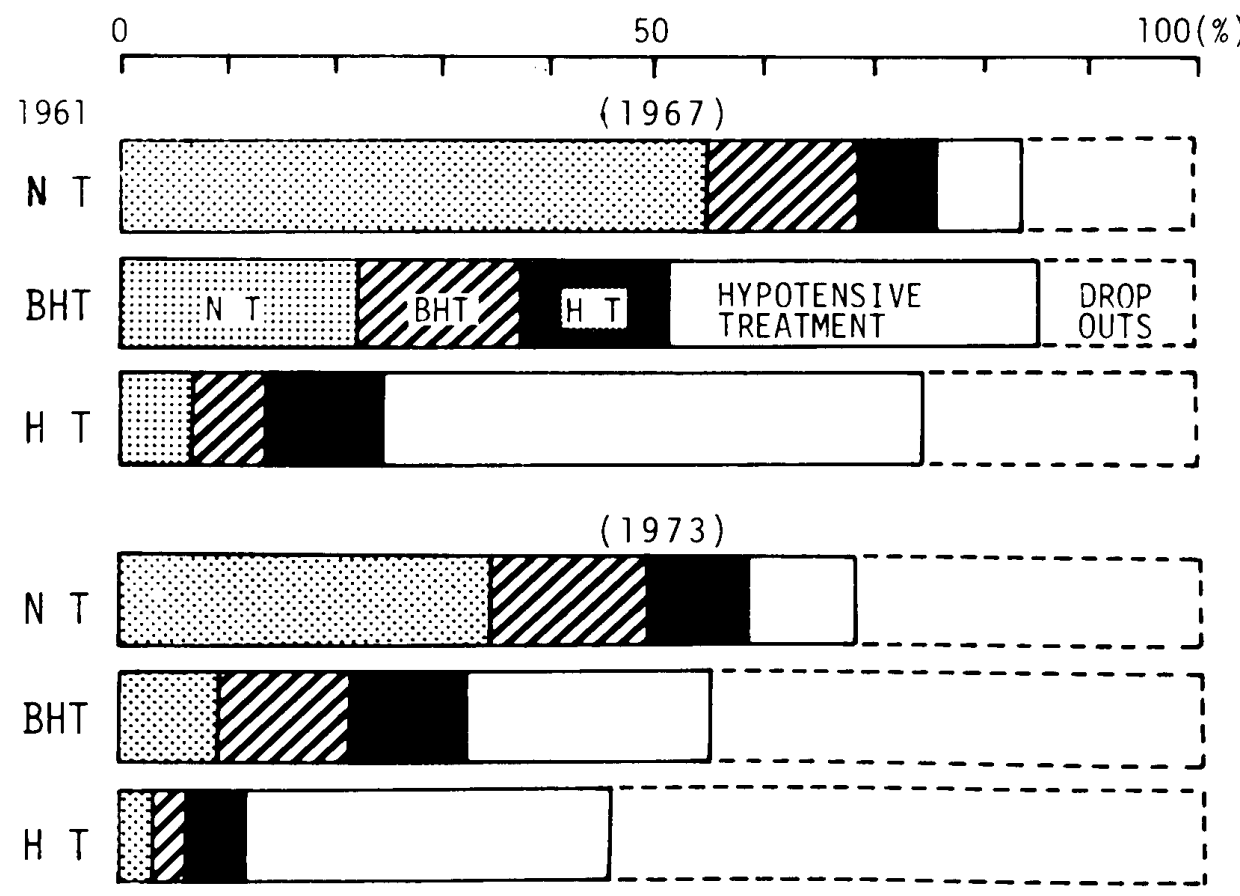

Fig.4. Changes in blood pressure status in blood pressure groups of both sexes during the periods of 1961-67 and 1961-73 in Hisayama. Sex and age were adjusted to the population in 1961.

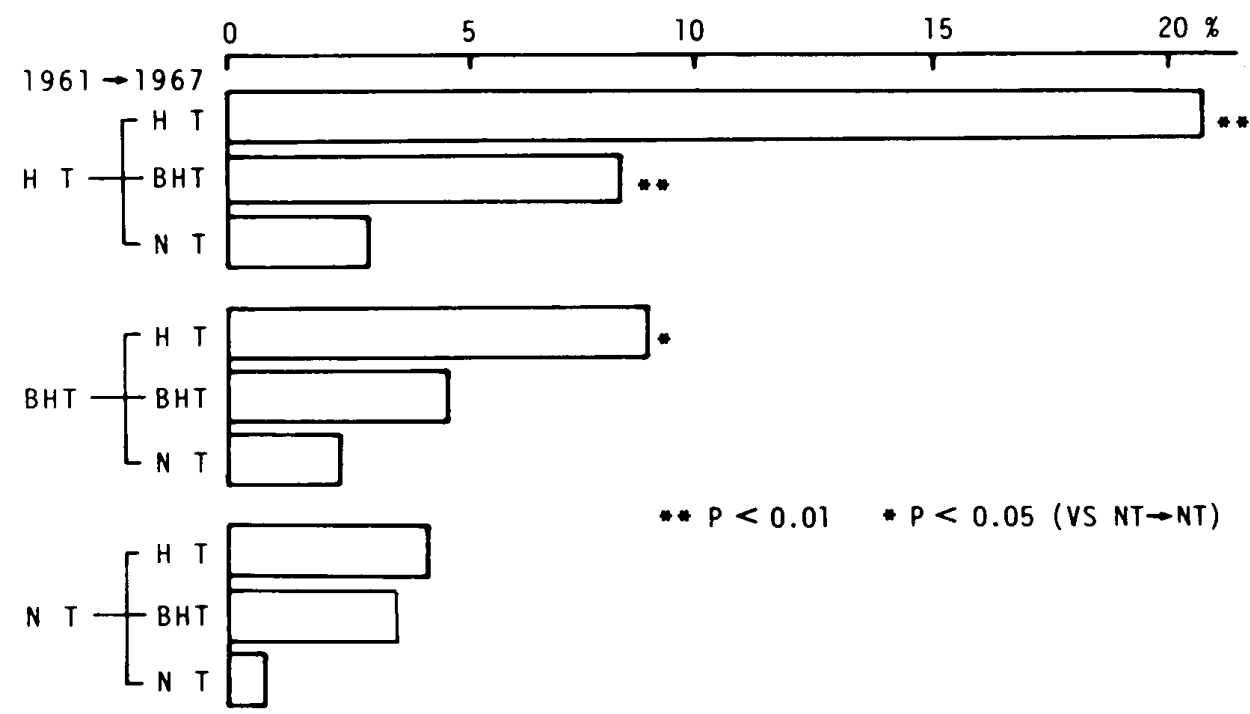

Fig.5. Cardiovascular mortality according to patterns.of change in blood pressure in both sexes in Hisayama (1967-79). Those taking hypotensive drugs were excluded. Age and sex were adjusted to the population in 1961 .

considered an important target of management.

The next question is whether or not the risk of death associated with hypertension is influ. enced by hypertensive organ damage, such as left ventricular hypertrophy, changes of ocular fundi or proteinuria. Figure 2 displays cardio- vascular mortality according to the presence or absence of hypertensive organ damage within the category of blood pressure level. Subjects with organ damage more frequently died from cardiovascular disease than those without in all categories of blood pressure except normotension in 
1. Stable Blood Pressure Groups

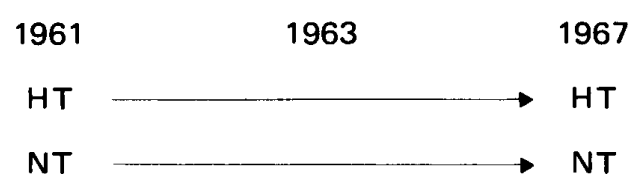

2. Labile Blood Pressure Groups

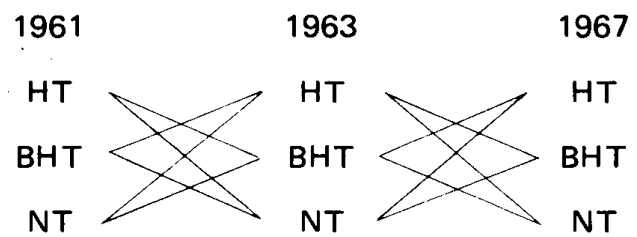

Fig.6. Patterns of change in blood pressure. $\mathrm{HT}=$ hypertension, NT = non-hypertension, $\mathrm{BHT}=$ borderline hypertension

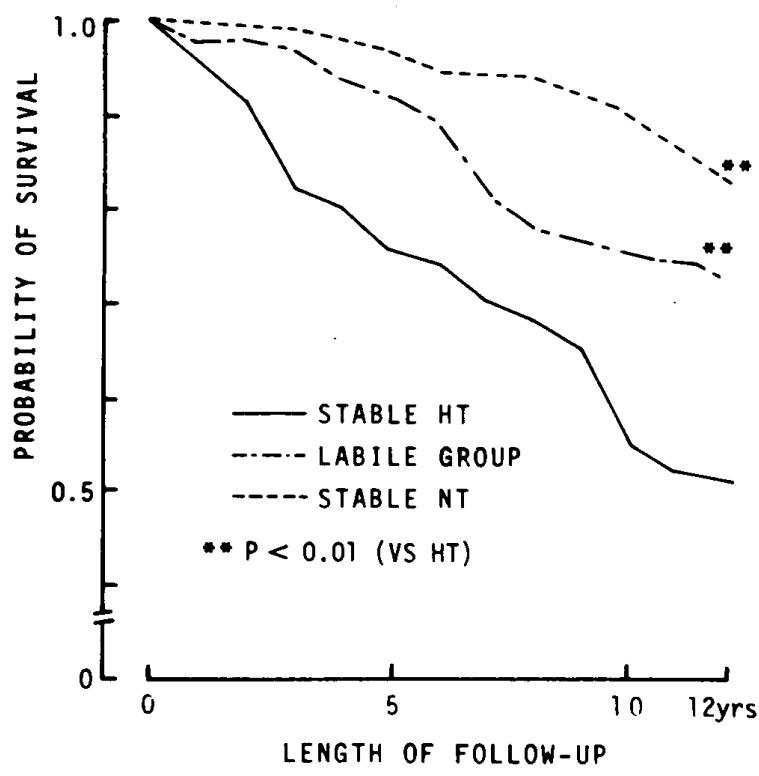

Fig.7. Age-adjusted survival by blood pressure groups in females in Hisayama (196779). $\mathrm{HT}=$ hypertension, $\quad \mathrm{NT}=$ nonhypertension.

females. Especially in males, this difference was significant in all categorical groups.

There is no generally accepted definition of what is the basal blood pressure, and moreover, it has been well supported that casual blood pressure at mass-screenings is important in assessing a long-term prognosis for the group of people. However, it is also true that in some patients the first recorded pressure may be situated at the very upper or lower range of their normal variation, and in others it may vary with physiologic stress influenced by circumstances and environment. The risk of mortality from cardiovascular disease related to systolic and diastolic blood pressure was estimated by both a single reading and an average of 3 readings of blood pressure at the time of entry. Figure 3 shows data only for females. SMR (standardized mortality ratio) is represented by the ratio of the observed to the expected number of cases which was computed by applying the age-sex specific mortality for the whole population to the various blood pressure subgroups under study. A mortality ratio of 1.0 indicates the average death rate due to cardiovascular disease for the whole population. As shown in Fig. 3, the difference of risk of both systolic and diastolic blood pressure levels was more noticeable in the mortality ratio caluculated by the average of 3 reading than that by a single reading. Assuming that the average value of the 3 readings is more similar to basal blood pressure, the mortality ratio for females exceeded the average of the whole population at the level of $80-89 \mathrm{mmHg}$ in diastolic blood pressure. Therefore, the threshold of the excess risk in diastolic blood pressure for females seemed to exist at approximately the level of 80 $\mathrm{mmHg}$.

\section{Longitudinal Observation of Blood Pressure and Cardiovascular Mortality}

Blood-pressure measurements of each subject were repeated at subsequent biennial examinations in an identical manner to that of the initial exam. This would provide not only an opportunity to relate a given blood pressure to disease development, but also to observe any longitudinal change in blood pressure. Examinations at 6-year intervals were selected to account for changes in the blood pressure of each individual. Thus, 3 examinations were used: at the time of entry, 6 th year and 12 th year. Since some of the subjects died or did not return for examination, the same identical cohort was not seen at each cycle. Figure 4 presents just the variation in classification of blood pressure - normotension, borderline, hypertension - at different examinations, namely between 1961 and 1967 or between 1961 and 1973. Without treatment for hypertension, a person classified as borderline at the time of entry was less frequently found to be borderline 6 or 12 years later. However, surprisingly, almost one-third of the borderline hypertensives among the Hisayama cohort had an experience of taking antihypertensive medications during these intervals.

Excluding those prescribed antihypertensive 
TABLE I PREVALENCE OF BORDERLINE HYPERTENSION IN HISAYAMA

\begin{tabular}{lcccc}
\hline \multirow{2}{*}{ Males } & & $\begin{array}{c}1961 \\
(n=1,621)\end{array}$ & $\begin{array}{c}1973-74 \\
(n=2,135)\end{array}$ & $\begin{array}{c}1978 \\
(n=2,449)\end{array}$ \\
\cline { 2 - 5 } & $H T$ & $26.7 \%$ & $20.7 \%$ & $16.8 \%$ \\
\hline \multirow{2}{*}{ Females } & BHT & 20.1 & 22.8 & 23.8 \\
\cline { 2 - 5 } & BHT & 24.2 & 23.2 & 19.6 \\
\hline
\end{tabular}

Age-adjusted to cohort population in 1961. HT =hypertension, BHT=borderline hypertension

TABLE II DIFFERENCES IN AGE, BLOOD PRESSURE AND PULSE RATES AT THE TIME OF ENTRY BETWEEN THOSE WITH STABLE BLOOD PRESSURE AND THOSE WHO DEVELOPED INTO HYPERTENSIVES

\begin{tabular}{|c|c|c|c|c|c|c|}
\hline & 1961 & 1967 & Age & $S B P$ & $D B P$ & Pulse rates \\
\hline \multirow{2}{*}{ Males } & \multirow{2}{*}{ Non-HT } & $H T$ & $55^{*}$ & $\begin{array}{l}137^{* *} \\
(150)\end{array}$ & $\begin{array}{l}77^{* *} \\
(86)\end{array}$ & 65 \\
\hline & & Non-HT & 52 & $\begin{array}{l}124 \\
(139)\end{array}$ & $\begin{array}{l}73 \\
(83)\end{array}$ & 62 \\
\hline \multirow{2}{*}{ Females } & \multirow{2}{*}{ Non-HT } & $H T$ & $58 * *$ & $\begin{array}{c}139 * * \\
(153)\end{array}$ & $\begin{array}{l}79 * * \\
(88)\end{array}$ & $71^{* *}$ \\
\hline & & Non-HT & 53 & $\begin{array}{l}125 \\
(139)\end{array}$ & $\begin{array}{l}73 \\
(82)\end{array}$ & 65 \\
\hline
\end{tabular}

\footnotetext{
( ): upper limits

$H T=$ hypertension, $S B P=$ systolic blood pressure,

$D B P=$ diastolic blood pressure, ${ }^{* *} p<0.01,{ }^{*} p<0.05$ (vs Non-HT)
}

drugs, the blood pressure of each individual at the time of entry was compared to the subsequent blood pressure at the 6-year interval (1967), and within each categorization of normotension, borderline and hypertension at the time of entry, later BP was classified into normotension, borderline and hypertension. This analysis does take into consideration any change that may have taken place in the blood pressure of the subjects over the 6-year period.

When the changing pattern of blood pressure was evaluated in terms of cardiovascular mortality for the subsequent 12-year period between 1967 and 1979, a clear dose-response relationship was apparent as indicated in Fig. 5. The age-sex adjusted mortality from cardiovascular disease went up from normotensive to borderline and to hypertensive classified in 1967 regardless of BP categorization at the time of entry. The highest mortality was observed in subjects whose $\mathrm{BP}$ was sustained in the hypertensive range during the 6-year period, and next in order were those with BP elevated from borderline to hyper- tensive, or those whose BP remained in borderline even though their BP showed a lowering trend.

\section{Mortality from Cardiovascular Disease according} to Stability or Instability of Blood Pressure

Since blood pressure has a labile nature, the term "labile hypertension" may be difficult to use with accuracy. However, mildly raised blood pressure often disappears and again appears in the longitudinal observation. In order to evaluate whether or not variability of blood pressure influences the life expectancy of the population, the blood pressure of each subject was analyzed in more detail at 3 examinations over the 6-year follow-up as shown in Fig. 6 . The stable BP group included subjects whose BPs were constantly classified as hypertension or normotension at 3 different examinations. On the other hand, the labile BP group consisted of those with BPs elevating or lowering from one category to another at 3 points of time.

Age-adjusted survival over the subsequent 12 
years for females according to groups classified by variation of blood pressure is shown in Fig. 7 . Among subjects whose BP persisted in the hypertensive range at all examinations (stable HT) less survived at any length of follow-up. More subjects in the stable NT group survived as compared to the labile one, but the survival rate of the latter was more favorable than that of stable HT $(\mathrm{p}<0.01)$.

Therefore, a clinical concept of labile hypertension in the broad sense does not seem to be so important for demonstrating a tendency toward the development of a cardiovascular catastrophe.

\section{Borderline Hypertension as a Predictor for Fu- ture Hypertension}

Certain questions can be explored from the longitudinal data of blood pressure. For instance, is it possible to predict who will develop hypertension? Has borderline hypertension any relation to the development of hypertension? It has long been believed that those persons who have rather low blood pressure are less inclined to develop hypertension. Again, the blood pressure of each subject in 1961 was compared to that in 1967, and the cohort population was divided into 2 groups: one included those with BP remaining in the non-hypertensive range; the other, those whose BP elevated from non-hypertensive in 1961 to hypertensive in 1967. Logistic discriminant analysis was applied to these 2 groups using 9 relevant variables determined at the time of entry, such as age, mean BP, diabetes mellitus, abnormal ECG findings (Minnesota Code III $_{1}$ ), ocular fundi more than KW II, proteinuria, pulse rates, Quetelet index and alcohol consumption. Contributing factors to discriminate those who developed hypertension from those who remained in non-hypertensive were only mean BP in males, and age, mean BP and pulse rate in females. Therefore, it was considered that the initial blood pressure is important in predicting whether or not the subjects will develop hypertension.

Average values of age, systolic and diastolic BP and pulse rate at the time of entry were calculated according to sex in 2 groups of BP category (Table II). Systolic BP in those who developed hypertension was $137 \mathrm{mmHg}$ in males and 139 in females, which was close to the cutoff point of borderline hypertension. Thus, mildly raised blood pressure itself may be a predictor of the development of hypertension in later life.

\section{DISCUSSION}

The prevalence of borderline hypertension has been studied in several population surveys. Julius and Schork ${ }^{7}$ have pointed out that the prevalence of borderline hypertension varies with numerous interacting factors, i.e., age, definition of the condition, sex, anthropometric measures, number and method of blood pressure readings. Taking those into account they summarized data found in the literature as follows: 1) The prevalence of systolic borderline hypertension in all ages above 20 is $10 \%$ or more. 2) Diastolic borderline hypertension is less than systolic. 3) Prevalence of borderline hypertension appears to increase with age. 4) Prevalence of borderline hypertension in females below 50 years old is smaller than in males. The different distribution between this study and others may depend on the disparity of characteristics and conditions of the population.

On the other hand, from epidemiological studies over the years there was evidence to suggest that a rough classification into normotension, hypertension and borderline would be valuable to estimate the prognosis of this disorder. However, some of them, particularly the results from the Framingham study, 8,9 have shown that the risk of disease and death from cardiovascular lesions increase in a graded fashion as the blood-pressure level rises. If it is true, the definition of hypertension may be arbitrary, and the limits between hypertension and borderline or borderline and normotension may be unclear. Therefore, when the significance of blood pressure raised mildly is evaluated in the epidemiological aspects, the following points must be considered: On how many measurements of blood pressure was it based? Does 'mild' refer only to pressure level or, to a lesser degree, to organ damage? The present study showed that blood-pressure level had a strong relationship with cardiovascular mortality even though it was based on a single casual blood pressure. When an evaluation was made using the average value of 3 blood pressure measurements, the relationship of blood pressure to mortality from cardiovascular disease became more striking. Hypertensive organ damage was also observed in borderline hypertensives and cardiovascular death was more frequent in borderline with organ damage than in those without. From these points of view, attention should be paid to the variation in blood pressure of borderline hypertensives. Julius ${ }^{10}$ 
had concluded that the majority of patients below 50 years old with borderline hypertension did not develop hypertension. However, borderline hypertension in younger subjects may be different from that in middle to old age with respect to the mechanism of elevating blood pressure and the prognosis. Our results showed that subjects with BP elevated from the boderline to hypertensive range would subsequently have a higher mortality from cardiovascular disease. The majority of those might be suffering from hypertensive organ involvement. On the contrary, a mere variation in blood pressure (labile BP) could not be correlated with a high mortality. The Manitoba study ${ }^{11}$ showed that the correlation coefficients between blood pressure measurements from 5 to 30 years apart were generally high and highly significant. Based on the present study, it would appear that the initial blood pressure is important in determining whether or not the subjects will develop hypertension. Moreover, the average value of the initial systolic blood pressure of those who will develop hypertension almost reach the arbitrary cut-off point categorized as borderline. The significance of borderline hypertension could be derived from the fact that individuals with borderline hypertension would in part develop established hypertension in the future.

Equally important is that borderline hypertension is much more common than severely hypertensive disease in the general population. From the present study, if blood pressure in this category can be controlled successfully, a large number of cardiovascular deaths could be prevented, because population-attributable risk of borderline hypertension is high, especially in females. Thus, borderline hypertension should be cosidered as a meaningful public health problem.

\section{REFERENCES}

1. WHO Expert Comittee: Arterial hypertension and ischemic. heart disease: Preventive aspect. WHO Technical Report Series No. 231, Geneva, 1962

2. KATSUKI S, HIROTA Y: Current concept of the frequency of cerebral hemorrhage and cerebral infarction in Japan. In Cerebral Vascular Disease: 5 th Conference, ed by SIEKERT RG, WHISNANT JP, Grune \& Stratton, New York, 1966, p 99

3. OMAE T, TAKESHITA M, HIROTA Y: The Hisayama study and joint study on cerebrovascular diseases in Japan. In Cerebrovascular Diseases: 10 th Princeton Conference, ed by Scheinberg $P$ Raven Press, New York, 1976, p 255

4. OMAE T, UEDA K, KIKUMURA T, SHIKATA T, FUJII I, YANAI T, HASUO Y: Cardiovascular death among hypertensive subjects of middle to old age: A long-term follow-up study in a Japanese community. In Hypertension in the Young and Old, ed by ONESTI G, KIM KE, Grune \& Stratton, New York, 1981, p 785

5. OMAE T: Hypertension among Hisayama residents. Jap J Hypertension 3: 101, 1981 (in Japanese)

6. DEUBNER DC, TYROLER HA, CASSEL JC, HAMES CG, BECKER C: Attributable risk, population attributable risk, and population attributable fraction of death associated with hypertension in a biracial population. Circulation 52: 901,1975

7. JULIUS S, SCHORK MA: Borderline hypertension: A critical review. J Chron Dis 23: 723, 1971

8. KANNEL WB: Some lessons in cardiovascular epidemiology. Am J Cardiol 37: 269, 1976

9. KANNEL WB: Role of blood pressure in cardiovascular disease: The Framingham study. $A n$ giology 26: 1975

10. JULIUS S: Borderline hypertension: An overview. Med Clin Nor Am 61: 495, 1977

11. RABKIN WS, MATHEWSON MB, TATE RB: Relationship of blood pressure in 20-30-year-old men to subsequent blood pressure and incidence of hypertension over a 30-year observation period. Circulation 65: 291, 1982 